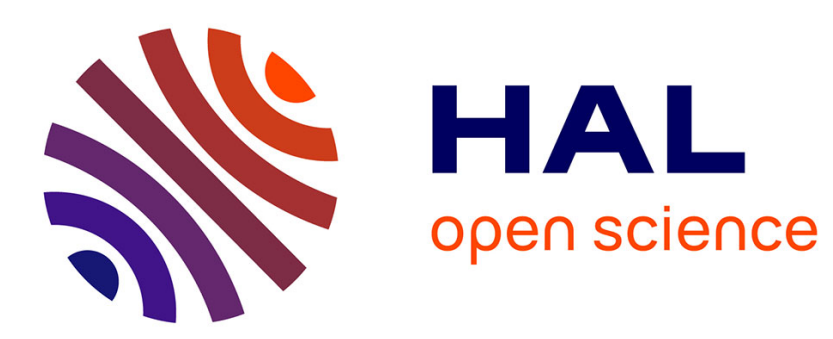

\title{
1-D Drift-Diffusion Simulation of Two-Valley Semiconductors and Devices
}

Markus Muller, Philippe Dollfus, Michael Schroter

\section{To cite this version:}

Markus Muller, Philippe Dollfus, Michael Schroter. 1-D Drift-Diffusion Simulation of Two-Valley Semiconductors and Devices. IEEE Transactions on Electron Devices, 2021, 68 (3), pp.1221-1227. 10.1109/TED.2021.3051552 . hal-03332527

\section{HAL Id: hal-03332527 \\ https://hal.science/hal-03332527}

Submitted on 2 Sep 2021

HAL is a multi-disciplinary open access archive for the deposit and dissemination of scientific research documents, whether they are published or not. The documents may come from teaching and research institutions in France or abroad, or from public or private research centers.
L'archive ouverte pluridisciplinaire HAL, est destinée au dépôt et à la diffusion de documents scientifiques de niveau recherche, publiés ou non, émanant des établissements d'enseignement et de recherche français ou étrangers, des laboratoires publics ou privés. 


\title{
1D Drift-Diffusion Simulation of Two-Valley Semiconductors and Devices
}

\author{
Markus Müller ${ }^{1}$, Philippe Dollfus ${ }^{2}$, Michael Schröter ${ }^{1}$
}

\begin{abstract}
A two-valley formulation of $1 D$ drift-diffusion transport is presented that takes the coupling between the valleys into account via a new approximation for the non-local electric field. The proposed formulation is suitable for the simulation of III-V heterojunction bipolar transistors as opposed to formulations that employ the single electron gas approximation with a modified velocity-field model, which also causes convergence problems. Based on Boltzmann transport equation simulations, model parameters of the proposed two-valley formulation are given for GaAs, InP, InAs and GaSb at room temperature. Applications of the new formulation are also demonstrated.
\end{abstract}

Index Terms - III-V semiconductors, drift-diffusion, charge carrier transport, negative differential mobility, TCAD, HBT.

\section{INTRODUCTION}

Transistors based on III-V semiconductors provide high transit frequencies simultaneously with high breakdown voltages, owing to their higher low-field mobility and larger bandgap compared to silicon based transistors. The development of both process technology along with device design optimization and adequate physics-based compact models for technology deployment may benefit tremendously from numerical device simulation (a.k.a. technology computer aided design or TCAD) under the following conditions. (i) The associated TCAD tools provide a reasonably accurate prediction of the impact of structural changes on the electrical device characteristics. (ii) The computational effort enables obtaining useful results within reasonable time. Meeting these requirements is becoming increasingly difficult with the reduction of critical device dimensions and the use of materials that exhibit additional physical effects compared to silicon. Both of these attributes apply to III-V semiconductors and are the main reason for the lack of suitable TCAD tools in that field.

In contrast to silicon based devices, III-V based devices employ materials in which electron transport in more than one conduction band is relevant [1-3]. Transport in (typically) two valleys with quite different mobility is usually accounted for using the so-called single electron gas (SEG) approximation with a modified electron velocity-field equation

${ }^{1}$ Chair for Electron Devices and Integrated Circuits, Technical University Dresden, 01062 Dresden, Germany. This work has been partially supported by the EU project H2020-ECSEL project TARANTO and the German National Science Foundation (DFG SCHR695/14, /21).

${ }^{2}$ Université Paris-Saclay, CNRS, Centre for Nanoscience and Nanotechnology, 91120 Palaiseau, France that accounts for the negative-differential-mobility (NDM) effect [3-5]. This approach has been used in moment-based transport equations like the drift-diffusion (DD) and hydrodynamic transport formulations. Due to their computational efficiency, those formulations are being widely used by device simulators (e.g. [7-9]) in industry and academia. It has been shown though that the SEG approach does not provide satisfactory results for III-V devices for several reasons [10-13]. The NDM formulation of the mobility leads to severe convergence problems, prohibiting simulations in the operating region of interest for circuit applications $[6,11,14]$. Moreover, the SEG approach is questionable due to its physical basis, which has extensively been discussed in [15]. Fundamentally, such issues can be overcome by using the Boltzmann transport equation (BTE) (e.g., [16-18]) or more complicated quantum transport formalisms (e.g., [19]).

However, these approaches are not suitable for practical applications due to their far too large computational effort and resulting simulation times. Therefore, a self-consistent solution of moment-based transport equations is highly desirable due to their far lower computational cost and parameters that are easier to adjust to measured device characteristics. Despite the fact that their physical basis at small length scales becomes questionable, it has been shown that even the DD approach can still be employed if the mobility is properly adjusted [20]. This insight is very beneficial for maintaining a basic analytical understanding of the major transport effects in a device. In particular, the simplicity of the DD transport formalism preserves the bridge between BTE and physics-based compact models for circuit design, since all mainstream compact models for FETs and HBTs are based on 1D DD transport (e.g. [21, 22]).

It is thus desirable to overcome the limitations of the SEG approximation within the DD transport formalism. In [6], a two-valley DD approach was proposed that uses a carrier temperature calculated from electrothermal considerations but still assumes a SEG. It has already been shown that two-valley hydrodynamic transport with two separate electron gas systems may be used to accurately reproduce BTE results [23]. However, the system in [23] requires a large number of parameters and is, in our opinion, not practical for the analysis and optimization of transistors that have both a spatially dependent material composition and doping density, such as heterojunction bipolar transistors (HBTs). In this work a two-valley (2v) formulation of 1D DD transport with two separate electron gas systems is proposed as a compromise between physical rigor and suitability for practical use of TCAD for device design, optimization and modeling. Section II introduces the rationale and physical basis for the $2 \mathrm{v}$-DD equation system, which is 
then formulated in section III. The application of the new $2 \mathrm{v}$ DD model is demonstrated in section IV.

The DD simulations shown herein have been obtained with the in-house simulator COOS [24], which has been extended for the simulation of III-V compound semiconductor heterojunction bipolar transistors with more than a single valley. All simulations herein have been conducted at $300 \mathrm{~K}$.

\section{FUNDAMENTALS}

The BTE can be used to model carrier transport on a microscopic scale in bulk semiconductors and devices $[16,17]$. The bulk velocity field characteristics of GaAs, InP, GaSb and InAs obtained from the BTE are shown in Fig. 1 along with measured data.

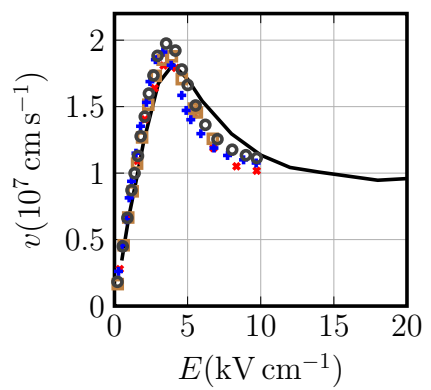

(a)

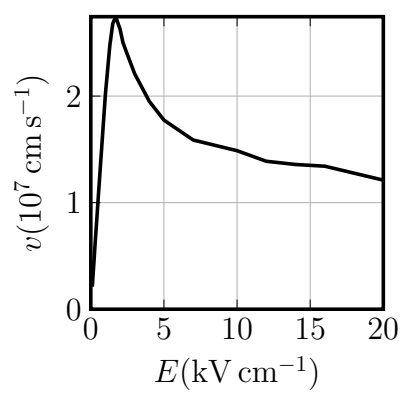

(c)

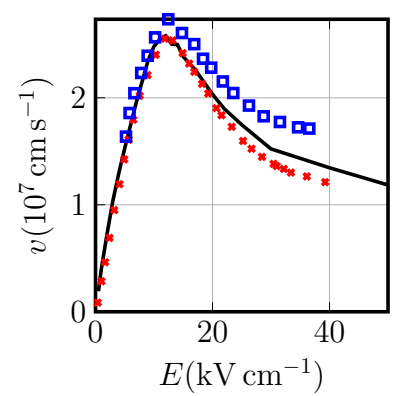

(b)

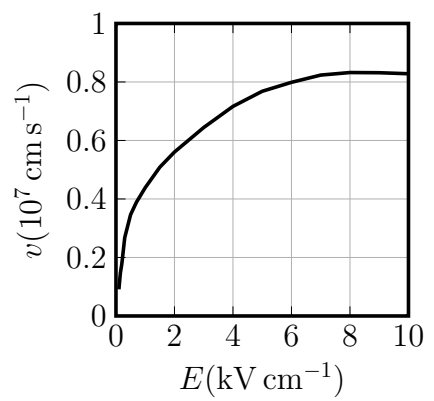

(d)
Fig.1. Measured (symbols) and simulated (solid lines) bulk velocity field characteristics of intrinsic (a) GaAs [25], (b) InP [26], (c) InAs and (d) $\mathrm{GaSb}$ at $300 \mathrm{~K}$ from the BTE. Note that no experimental data for (c) and (d) have been found for $300 \mathrm{~K}$.

For a semiconductor with several valleys one may use the method of moments to derive a system of equations from the BTE [15]. Conservation of particles is expressed as

$$
\frac{\mathrm{d} n_{j}}{\mathrm{~d} t}+\frac{\mathrm{d}\left(v_{j} n_{j}\right)}{\mathrm{d} x}=\left(\frac{\mathrm{d} n_{j}}{\mathrm{~d} t}\right)_{\mathrm{c}},
$$

where $x$ is the transport direction, $t$ is the time, $n_{\mathrm{j}}$ is the carrier density and $v_{\mathrm{j}}$ is the carrier velocity in valley $j$. The collision term in (1), denoted with subscript $c$, is of crucial importance for the simulation of many-valley semiconductors. It describes the coupling between the valleys due to scattering, including thermal carrier generation and recombination.

For the two valley $(j=\Gamma, \mathrm{L})$ equation system, numerical solutions based on the hydrodynamic and energy-transport approximations outlined in [15] have been obtained in [23, 27]. However, to the best of our knowledge, such formulations have not been implemented in any open-source or commercial TCAD simulator. This may be related to practical issues. First, the implementation and numerical effort is quite large. Second, the accuracy compared to measurements is not necessarily improved when more moments of the BTE are included, since more moments require more parameters that also need to be accurately determined $[28,29]$. If predictive device simulations are required, the BTE should be solved [28].

\section{MODEL FORMULATION}

\section{A. Two-Valley Drift Diffusion Model}

To reduce the computational effort, the analysis is restricted to just the most important valleys. For the practically relevant III-V semiconductors, transport in the $\Gamma$ and the $\mathrm{L}$ valley dominates for electric fields typically encountered in device and circuit applications. To be on the safe side, the BTE simulations used as references in this work also include the $\mathrm{X}$ valleys. The $\mathrm{L}$ valley results shown herein are the averages over the simulated $\mathrm{L}$ and $\mathrm{X}$ valleys.

From (1) and $j=\Gamma, \mathrm{L}$, one gets two continuity equations

$$
\frac{\mathrm{d} n_{j}}{\mathrm{~d} t}=\frac{1}{\mathrm{q}} \frac{\mathrm{d} J_{\mathrm{n} j}}{\mathrm{~d} x}+\left(\frac{\mathrm{d} n_{j}}{\mathrm{~d} t}\right)_{\mathrm{c}},
$$

where

$$
J_{\mathrm{n} j}=-\mathrm{q} \mu_{\mathrm{n} j}\left(w_{j}\right) n_{j} \frac{\mathrm{d} \varphi_{\mathrm{n} j}}{\mathrm{~d} x} .
$$

Here $J_{\mathrm{n} j}$ is the current density, q is the elementary charge, $\mu_{\mathrm{n} j}$ is the carrier mobility, $\varphi_{\mathrm{n} j}$ is the quasi-Fermi potential (QFP) and $w_{j}$ is the carrier energy in valley $j$. This formulation of the DD current density is better suited for the analysis of heavily doped devices compared to Blotekjaer's formulation and can also be derived directly from the BTE [30]. For a 2v-DD equation system the collision terms can be written as

$$
\left(\frac{d n_{j}}{d t}\right)_{\mathrm{c}}=-n_{j} f_{j k}\left(w_{j}\right)+n_{k} f_{k j}\left(w_{k}\right)
$$

where $j=\Gamma$ when $k=\mathrm{L}$ and vice-versa. The functions $f$ are the intervalley transition frequencies that describe the intervalley scattering processes as a function of $w_{\mathrm{j}}$ and $w_{\mathrm{k}}$. The collision terms cause the energy dependence of the carrier populations among the valleys. The intervalley transition frequencies can be obtained from BTE simulations as explained in [16].

Since the carrier energies are not directly available in a DD formulation, $\mu_{\mathrm{n} j}$ and $f_{j k}$ are modeled as functions of the absolute value of a driving force $F_{j}$. This force needs to be calculated from local quantities and is used to estimate the carrier energy in each valley. For a homogeneous bulk semiconductor the carrier energy is a function of the absolute value of the electrical field $E$ [31]. This allows to find empirical model equations for $\mu_{\mathrm{n} j}$ and $f_{j k}$ as functions of $E$ from bulk BTE simulations. In non-homogeneous structures other fields are more suitable as driving forces, as will be discussed in Sec. IV.

\section{B. Intervalley Transfer Rate Model}

According to Fig. 2(a), $f_{\Gamma \mathrm{L}}$ shows a distinct dependence on the electric field, which is modeled by 


$$
f_{\Gamma \mathrm{L}}(|E|)=f_{\Gamma \mathrm{L}, \mathrm{equ}}+a_{f} \ln \left(1+\exp \left(\frac{|E|-E_{\mathrm{ivt}}}{E_{f \Gamma L}}\right)\right),
$$

where $a_{\mathrm{f}}$ is a model parameter and $E_{\mathrm{f \Gamma L}}$ is a normalization constant $(=1 \mathrm{kV} / \mathrm{cm})$. The critical field for the onset of intervalley transfer $E_{\mathrm{ivt}}$ is modeled doping dependent as

$$
E_{\mathrm{ivt}}=E_{\mathrm{ivt}, 0}\left[1+\left(\frac{N_{\mathrm{I}}}{N_{\mathrm{ivt}}}\right)^{\zeta_{\mathrm{ivt}}}\right] \text {, }
$$

where $E_{\mathrm{ivt}, 0}, N_{\mathrm{ivt}}$ and $\zeta_{\mathrm{ivt}}$ are model parameters. In equilibrium, (4) yields the relationship

$$
f_{\Gamma \mathrm{L}}(|E|=0)=\frac{n_{\mathrm{L}, \mathrm{equ}}}{n_{\Gamma, \text { equ }}} f_{\mathrm{L} \Gamma}(|E|=0),
$$

with $n_{\Gamma(\mathrm{L}) \text {,equ }}$ as the equilibrium carrier concentration in the $\Gamma(\mathrm{L})$ valley. The parameter $f_{\Gamma \mathrm{L} \text {,equ }}$ in (5) is implicitly determined by the band structure, (7) and by the model for $f_{\mathrm{L} \Gamma}$, which is introduced next.

The results in Fig. 2(b) indicate that $f_{\mathrm{L} \Gamma}$ is almost independent of doping but a linear function of the field, hence

$$
f_{\mathrm{L} \Gamma}(|E|)=f_{\mathrm{L} \Gamma 0}+\delta_{f}|E| .
$$

The field dependence of $f_{\mathrm{L} \Gamma}$ has only minor influence on the distribution of carriers amongst the valleys, since the dynamic range of $f_{\Gamma \mathrm{L}}$ is much larger. The noise observed in the simulated $f_{\mathrm{L} \Gamma}$ results from the very small amount of carriers in the $L$ valley at low fields.

\section{Velocity Formulation}

The carrier velocity in each band as a function of the electric field is shown in Fig. 3 for GaAs. The velocity field curve for carriers in the $\Gamma$ valley shows a slight NDM effect for doping concentrations below about $5 \times 10^{16} \mathrm{~cm}^{-3}[32,33]$, which has not been included in this work for the following reasons. (i) The low impurity concentrations where this effect occurs are of little relevance in advanced high-speed HBTs. (ii) For field values where this region would be relevant, the amount of carriers in the $\Gamma$ valley is small. Neglecting this effect causes minor deviations between BTE and the $2 \mathrm{v}$-DD model at high fields.

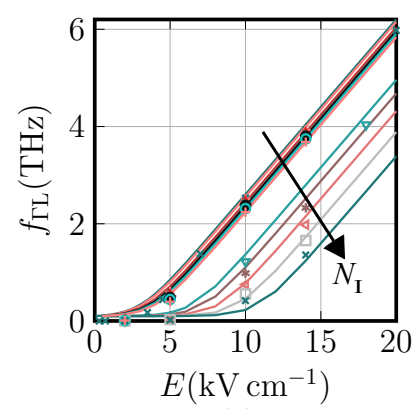

(a)

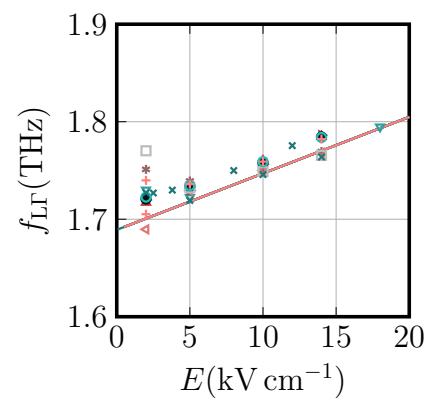

(b)
Fig.2. Intervalley transition frequency in GaAs for $N_{\mathrm{I}}=\left[1.0 \times 10^{15}\right.$, $1.4 \times 10^{16}, 2.2 \times 10^{16}, 3.3 \times 10^{16}, 5.2 \times 10^{16}, 8.0 \times 10^{16}, 1.1 \times 10^{18}$, $1.7 \times 10^{18}, 2.7 \times 10^{18}, 4.2 \times 10^{18}, 6.4 \times 10^{18} \mathrm{~cm}^{-3}$. Comparison between BTE (symbols) and analytical approximations (lines) for (a) $f_{\Gamma \mathrm{L}}$ with (5) and (b) $f_{\mathrm{L} \Gamma}$ with (8).

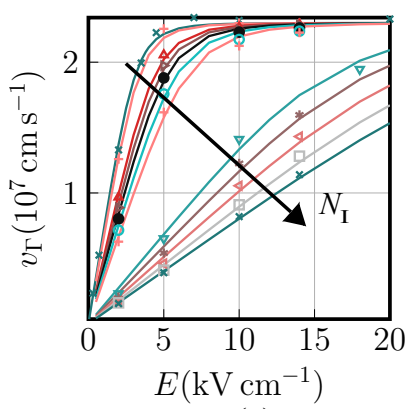

(a)

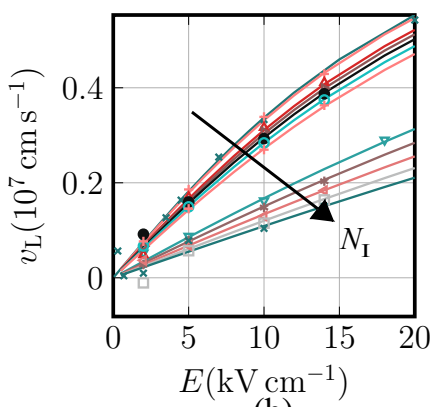

(b)
Fig.3. Average velocity in (a) the $\Gamma$ and (b) the $L$ valley of GaAs from BTE (symbols) and from (10) (lines) for the same impurity concentrations $N_{\mathrm{I}}$ as listed in Fig. 2.

The mobility model proposed herein has separate parameters for each valley $j$. It consists of a low-field mobility due to lattice and impurity scattering [34]

$$
\mu_{j, \mathrm{LI}}=\mu_{j, \min }+\frac{\mu_{j, \mathrm{~L}}-\mu_{j, \min }}{1+\left(\frac{N_{\mathrm{I}}}{N_{j, \text { ref }}}\right)^{\alpha_{j}}},
$$

where $\mu_{j, \mathrm{~L}}$ is the lattice mobility, $\mu_{j, \min }$ is the mobility at high impurity concentrations and $\alpha_{j}$ and $N_{j, \text { ref }}$ are model parameters. Finally, the mobility field dependence is given as

$$
\mu_{\mathrm{n} j}=\frac{\mu_{j, \mathrm{LI}}}{\left[1+\left(\frac{\mu_{j, \mathrm{LI}}|E|}{v_{j, \mathrm{sat}}}\right)^{\beta_{j}}\right]^{1 / \beta_{j}}}
$$

with $v_{j, \text { sat }}$ as the saturation velocity and $\beta_{j}$ as a model parameter. This mobility model is available in this or a similar form in most TCAD simulators.

\section{MODEL APPLICATION}

\section{A. Steady-State Bulk Velocity-Field Characteristics}

The average carrier velocity in a homogeneous bulk semiconductor with two valleys is

$$
v=\frac{n_{\mathrm{L}} v_{\mathrm{L}}+n_{\Gamma} v_{\Gamma}}{n_{\mathrm{L}}+n_{\Gamma}},
$$

where $n_{\Gamma}+n_{\mathrm{L}}=N_{\mathrm{I}}$. Under steady-state conditions the 1.h.s. of (4) equals zero, resulting in

$$
\frac{n_{\mathrm{L}}}{n_{\Gamma}}=\frac{f_{\mathrm{L} \Gamma}(|E|)}{f_{\Gamma \mathrm{L}}(|E|)},
$$

which can be evaluated using (5) and (8) [35]. The parameters for the model equations of $f_{\mathrm{L} \Gamma}, f_{\Gamma \mathrm{L}}, \mu_{\mathrm{nL}}$ and $\mu_{\mathrm{n} \Gamma}$ have been obtained by fitting (5), (8) and (10) to BTE simulation results. The bulk velocity field characteristics are then obtained using (11) and (12). The comparison of the 2v-DD model with BTE data is shown in Fig. 4. Quite good agreement, including the NDM effect, is observed over a wide range of doping levels ${ }^{1}$. The model parameters are summarized in Appendix B. 


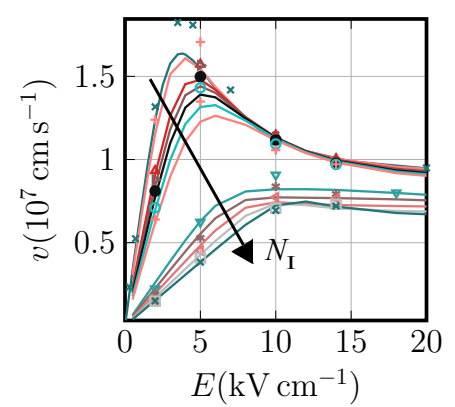

(a)

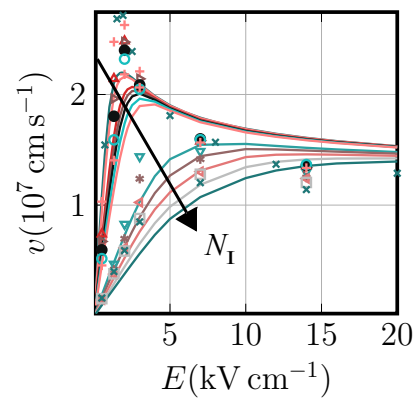

(c)

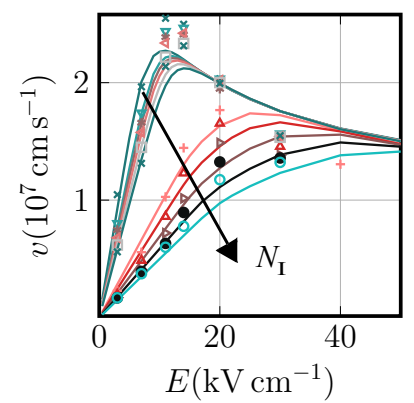

(b)

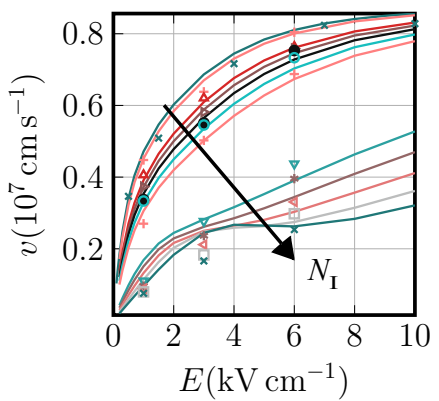

(d)
Fig.4. Bulk velocity-field characteristic of (a) GaAs, (b) InP, (c) InAs and (d) GaSb from BTE simulation (symbols) and the $2 \mathrm{v}-\mathrm{DD}$ equation system (lines) for the same impurity concentrations $N_{\text {I }}$ as listed in Fig. 2.

\section{B. Driving Force and Application to Test Structures}

In this section different choices for the driving force $F_{j}$, labeled (i)-(iii), are discussed using two n+-n-n+ test structures as examples. Common choices for driving forces are the gradients of the QFPs and the electric field. Approach (i) and (ii) are defined as:

$$
\begin{gathered}
F_{j}=\left|\frac{\mathrm{d} \varphi_{n j}}{\mathrm{~d} x}\right| \\
F_{j}=\left|\frac{\mathrm{d}}{\mathrm{d} x}\left(W_{j 0}-W_{j}\right)\right|
\end{gathered}
$$

(i) is often used in DD simulations and has been proven useful in practice [36]. Using (ii), the local energy in valley $j$ is estimated from $\mathrm{d}\left(W_{\mathrm{j} 0}-W_{\mathrm{j}}\right) / \mathrm{dx}$, where $W_{j}$ is the band edge of valley $j$ and the index 0 indicates its equilibrium value. Note that for a homogeneous bulk semiconductor, $\mathrm{d}\left(W_{\mathrm{j} 0^{-}} W_{\mathrm{j}}\right) / \mathrm{dx}$ is equal to the electric field ${ }^{2}$.

BTE results of the mean carrier energy, along with the gradients for (i) and (ii) are shown in Fig. 5. The QFPs in Fig. 5 have been extracted from the BTE assuming

$$
n_{j}=n_{\mathrm{i} j} \exp \left(\frac{\Psi-\varphi_{\mathrm{n} j}}{V_{\mathrm{T}}}\right)
$$

1. Due to the lack of published experimental data over a sufficiently wide doping concentration range (GaAs, $\mathrm{InP}$ ) or at all ( $\mathrm{GaSb}, \mathrm{InAs})$ BTE data have been used as reference for calibrating the DD model.

2. In the general $3 \mathrm{D}$ case the $\mathrm{d} / \mathrm{dx}$ operators in (i) and (ii) need to be replaced with $\left(\vec{J}_{\mathrm{n} j} / \mid \vec{J}_{\mathrm{n} j}\right) \nabla$. as would be valid for DD transport; $n_{\mathrm{ij}}$ is the effective intrinsic carrier density of valley $j, \psi$ is the electrostatic potential and $\mathrm{V}_{\mathrm{T}}$ is the thermal voltage. All fields show a similar behavior and a significant deviation from the shape of the mean carrier energy. Thus, at least for this structure, the local fields are unsuitable for estimating the non-local energy acquisition.

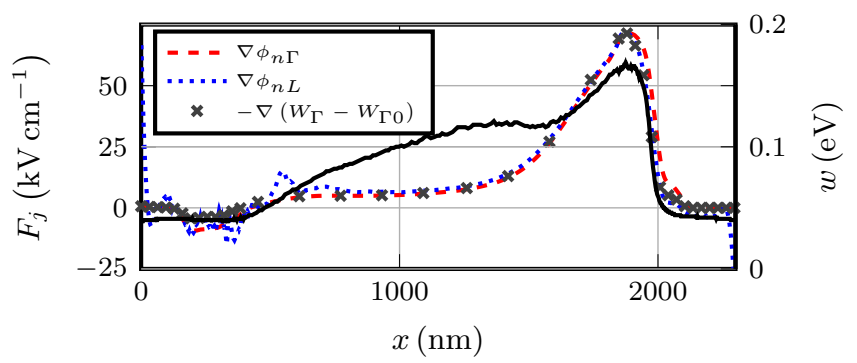

Fig.5. Comparison of different fields (left axis) based on local quantities and the mean carrier energy (right axis, black solid line) for the GaAs n+-n-n+ structure of Fig. 6. The applied voltage is $3 \mathrm{~V}$.

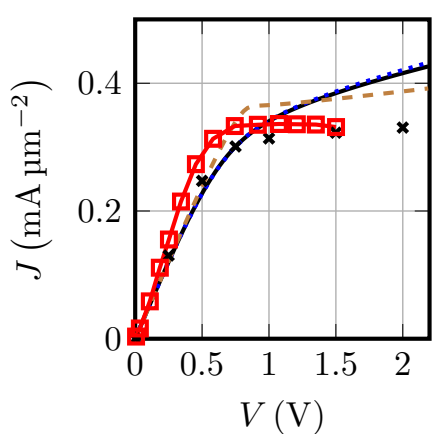

(a)

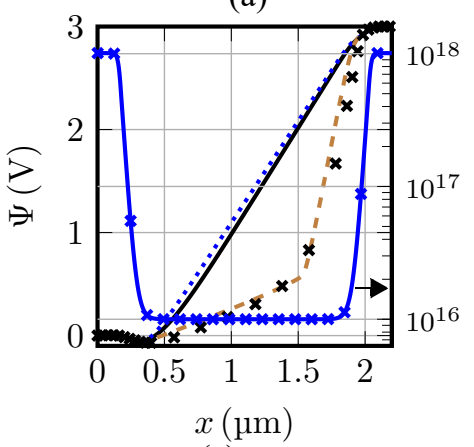

(c)

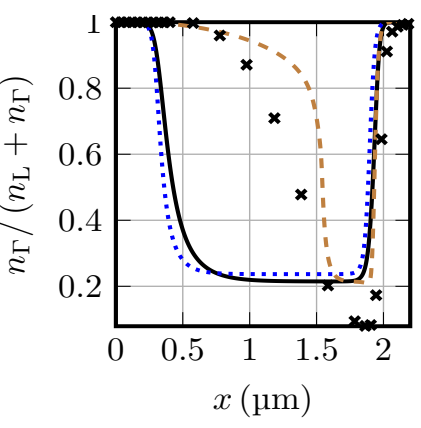

(b)
Fig.6. Comparison of (a) current density, (b) carrier distribution between the valleys and (c) electrostatic potential (left y-axis) and doping profile (blue solid line, right axis) of a $\mathrm{n}^{+} \mathrm{nn}^{+}$ GaAs structure. The bias in (b) and (c) is $V=3 \mathrm{~V}$. The choices (i)-(iii) for the driving force are described in the text.

In Fig. 6 simulations with (i) and (ii) are compared to measurement data and BTE simulations. In both cases, the velocity saturation in the $\Gamma$ valley had to be turned off to achieve convergence with (i) and (ii). As expected, both choices are unable to reproduce the BTE results satisfactorily and, thus, to describe non-local effects within the DD transport framework. To overcome this limitation, another driving force needs to be found. This driving force is used in approach (iii) and will be introduced, next. 
Before (iii) is derived, the principal properties between energy and electric field are discussed based on the BTE results in Fig. 7. Regardless of the bias level, the energy peaks on the right side of the n-layer. Even though the field is almost constant for low bias, the energy shows a spatial dependence. At high bias, the mean energy tends to saturate around $0.12 \mathrm{eV}$, while the field is still significantly increasing.

To account for the non-local energy-field dependence, the local field needs to be transformed into an effective local field such that the local energy is estimated correctly. As shown in Appendix A, a simplified energy balance equation for the $\Gamma$ valley reads

$$
\frac{\mathrm{d}}{\mathrm{dx}}\left(-\frac{5}{2} \frac{k_{\mathrm{B}} T_{\Gamma}}{\mathrm{q}} J_{\mathrm{n} \Gamma}\right)=-J_{\mathrm{n} \Gamma} \frac{\mathrm{d} \varphi_{\mathrm{n} \Gamma}}{\mathrm{d} x}-\frac{3}{2} k_{B} n_{\Gamma} \frac{T_{\Gamma}-T_{0}}{\tau_{w}} .
$$

Here, $\tau_{\mathrm{w}}$ is the energy relaxation time, $T_{0}$ is the lattice temperature and $T_{\Gamma}$ is the average carrier temperature in the $\Gamma$ valley. Near steady-state, (14) allows defining an effective local field

$$
\left|\frac{\mathrm{d} \varphi_{\mathrm{n} \Gamma, \mathrm{eff}}}{\mathrm{d} x}\right|=\frac{5 k_{\mathrm{B}}}{2 \mathrm{q} \lambda_{\Gamma}}\left(T_{\Gamma}\left(x, \mid \frac{\mathrm{d} \varphi_{\mathrm{n} \Gamma}}{\mathrm{d} x}\right)-T_{0}\right),
$$
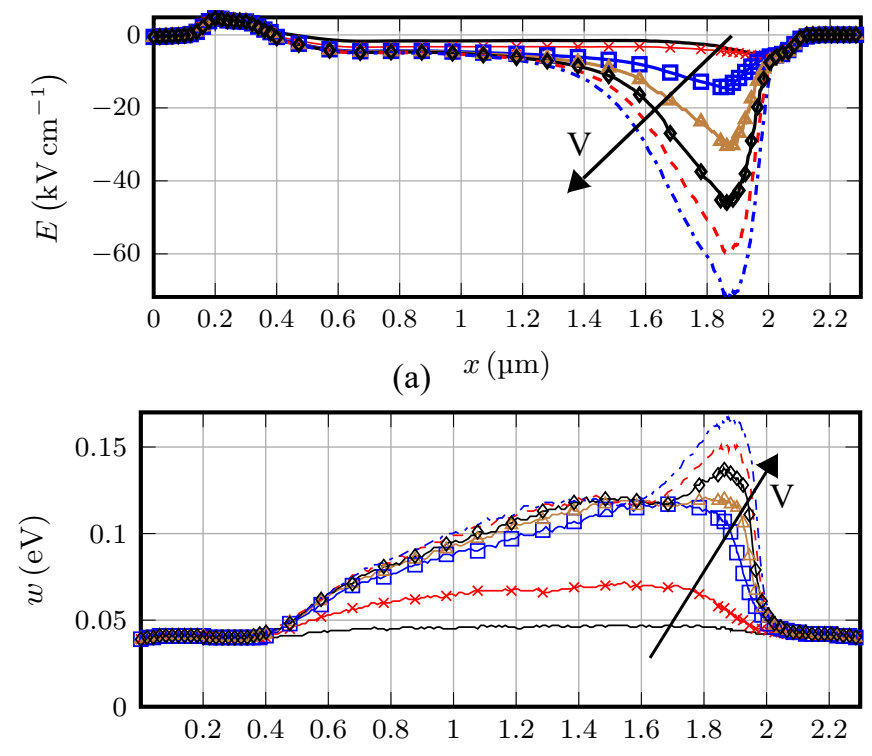

(b) $x(\mu \mathrm{m})$

Fig.7. (a) Electric field and (b) mean carrier energy from BTE simulations of the $\mathrm{n}^{+} \mathrm{nn}^{+} \mathrm{GaAs}$ structure for different applied voltages $0.25-3 \mathrm{~V}$.

where $\lambda_{\Gamma}$ is a parameter related to the energy relaxation time and the saturation velocity (see Appendix A). In regions where carrier heating is relevant, but intervalley transfer is not, a rough solution of (14) may be obtained by assuming that the field is spatially constant and that carriers travel at saturation velocity in the transport direction $x$. Then, the solution of (14) is [37]

$$
T_{\Gamma}\left(x, \frac{\mathrm{d}\left|\varphi_{\mathrm{n} \Gamma}\right|}{\mathrm{d} x}\right)=T_{0}+\frac{2 \mathrm{q}}{5 k_{\mathrm{B}}}\left|\frac{\mathrm{d} \varphi_{\mathrm{n} \Gamma}}{\mathrm{d} x}\right| \int_{x_{\mathrm{b}}}^{x} \exp \left(\frac{\xi-x}{\lambda_{\Gamma}}\right) \mathrm{d} \xi,
$$

where $\lambda_{\Gamma}$ is used as a fitting parameter and $x_{\mathrm{b}}$ is the first point in the carrier heating region.

In $\mathrm{n}^{+}$regions, the gradient of the quasi-Fermi potential is negligible and hence $T_{\Gamma}$ equals $T_{0}$. In the $\mathrm{n}$ region (16) can be used to estimate $T_{\Gamma}$ as long as intervalley transfer is negligible. When $T_{\Gamma}$ becomes sufficiently large, intervalley transfer becomes relevant and an analytical solution for $T_{\Gamma}$ is difficult to obtain. From the BTE solutions in Fig. 7(b) one can deduce that the energy, and thus also $T_{\Gamma}$, is saturating beyond a certain critical field. Hence, a suitable driving force is

$$
F_{\Gamma}=\frac{\left|\mathrm{d} \varphi_{\mathrm{n} \Gamma, \mathrm{eff}} / \mathrm{d} x\right|}{\left[1+\left(\frac{\left|\mathrm{d} \varphi_{\mathrm{n} \Gamma, \mathrm{eff}} / \mathrm{d} x\right|}{E_{\text {crit }}}\right)^{\gamma}\right]^{1 / \gamma}},
$$

where $E_{\text {crit }}$ and $\gamma$ are used as fitting parameters and (15)-(16) are used to calculate $\mid \mathrm{d} \varphi_{\mathrm{n} \Gamma}$, eff $/ \mathrm{dx} \mid$. For the L valley it is assumed that

$$
F_{\mathrm{L}}=\left|\frac{\mathrm{d} \varphi_{\mathrm{L}}}{\mathrm{dx}}\right|,
$$

which overestimates the $\mathrm{L}$ valley energy. This error is not critical, since carriers transferred to the L valley travel near saturation velocity anyway. Furthermore, $f_{\Gamma \mathrm{L}}$ is only weakly dependent on $F_{\mathrm{L}}$. Equations (iii-a) and (iii-b) define approach (iii).

Approach (iii) leads to quite good agreement for the electrostatic potential and carrier densities as shown in Fig. 6(b), (c). Also, the terminal current density exhibits qualitatively better agreement with BTE and experimental data at high fields (see Fig. 8(a)), but is now somewhat too large. With a proper adjustment of the $\mathrm{L}$ valley saturation velocity, the agreement can be improved further. The proposed use of the effective field in (iii-a) provides a practical approach towards capturing negative different mobility effects in a DD transport framework. The simulation results for an InP $n+-n-n+$ structure, shown in Fig. 8, further underline the suitability of this approach to capture non-local effects.

\section{Application to GaAsSb/InP HBT}

Fig. 9 depicts simulation results for a GaAsSb/InP HBT [38], using the 2v-DD equation system. In addition to the two electron continuity equations (3) and the Poisson equation, a hole continuity and transport equation needs to be solved. Herein, the mobility model and corresponding parameters from [39] are used. At the abrupt heterojunction, a special boundary condition is required [39]. In the middle of the neutral base the hole quasi-Fermi potential is forced to equal the applied base voltage [40].

To estimate the non-local energy, approach (iii) is used, where the collector is treated in the same way as the $\mathrm{n}$ region before. The material properties of the GaAsSb alloy are calculated using the parameters of its constituents and assuming bowing parameters equal to zero. The solution shows similar trends as the results in $[17,23]$. In particular, the $\mathrm{L}$ valley carrier density in the $\mathrm{BC}$ space-charge-region increases (s. Fig. $9(b))$ and exceeds the $\Gamma$ valley carrier density with increasing bias. The impact of intervalley transfer on the transit frequency 
can be seen in Fig. 9(c), where simulation results without intervalley transfer are shown for comparison. As the current density increases, more carriers get transferred to the L valley, decreasing the transit time due to the lower velocity in that valley. A calibration of the model to measured data for this transistor is left for future work.

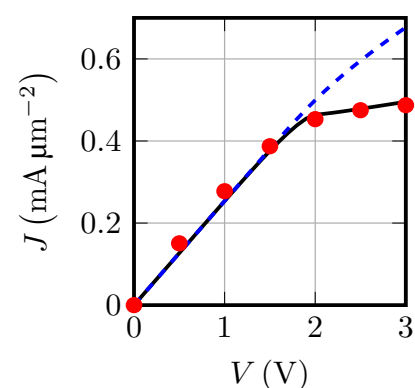

(a)

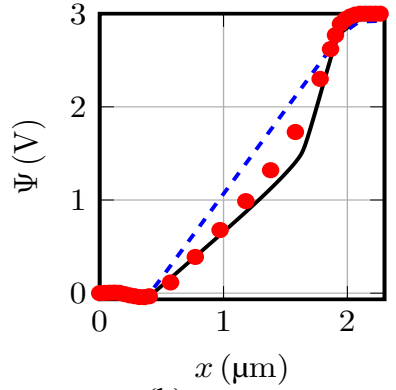

(b)

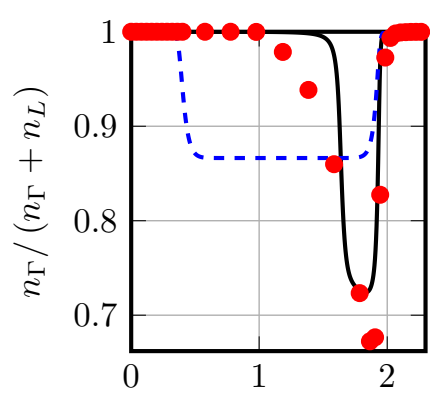

(c) $x(\mu \mathrm{m})$

Fig.8. Comparison of (a) current density, (b) electrostatic potential and (c) carrier distribution between the valleys of an InP structure with the same doping profile as depicted in Fig. 6(c) from BTE (dots) and DD simulations with the energy estimator approach (iii) (solid lines) and (i) (dashed lines). The bias in (b) and (c) is $V=3 \mathrm{~V}$.

\section{CONCLUSION}

A two-valley DD formulation for the simulation of III-V semiconductors has been developed. The transition of charge carriers between the two bands is described by a model for intervalley transfer that employs an effective electric field in order to capture the non-local energy acquisition of carriers. As opposed to standard single-valley transport models employed in most TCAD simulators, the new $2 \mathrm{v}$-DD transport formulation captures the negative differential mobility effect in a natural way while at the same time maintaining computational efficiency and limiting the number of model parameters.

The parameters for widely used III-V materials have been obtained from bulk BTE simulations at room temperature. Examples for the application of the new 2v-DD model have been presented. Future investigations will focus on the application of the two-valley DD model to III-V HBTs and compact modeling support. Also, it would be useful to determine bowing parameters for III-V alloys.

\section{Appendix A: Carrier temperature}

The energy-balance equation is given by (6) in [41]. It is also valid also for III-V semiconductors as long as intervalley transfer, and thus the associated energy flux, can be neglected. With the assumptions given in [42] and using (3), one can derive (14). Assuming $\mathrm{d} J_{\mathrm{n} \Gamma} / \mathrm{d} x=0$ and carriers traveling at saturation velocity allows to rewrite (14) as

$$
-\frac{5}{2} \frac{k_{\mathrm{B}} T_{\Gamma}}{\mathrm{q}} J_{\mathrm{n} \Gamma} \frac{\mathrm{d} T_{\Gamma}}{\mathrm{d} x}=-J_{\mathrm{n} \Gamma} \frac{\mathrm{d} \varphi_{\mathrm{n} \Gamma}}{\mathrm{d} x}-\frac{5}{2} k_{B} n_{\Gamma} v_{s \Gamma} \frac{T_{\Gamma}-T_{0}}{\lambda_{\Gamma}},
$$

where $v_{\Gamma, \text { sat }}$ is the $\Gamma$ valley saturation velocity and $\lambda_{\Gamma}=5 v_{\Gamma, \text { sat }} \tau_{\mathrm{w}} / 3$ is the energy relaxation length as defined in [37]. This equation allows to derive (15) and (16) [37].

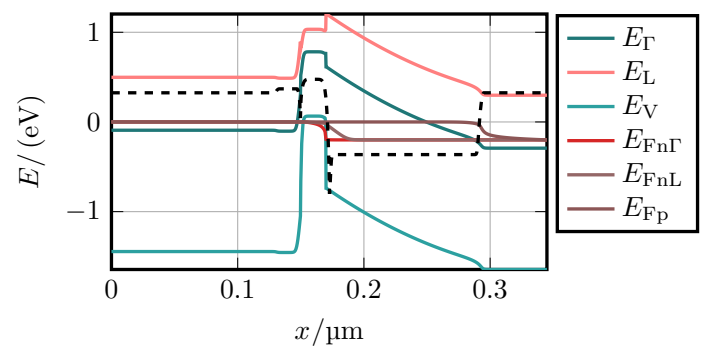

(a)

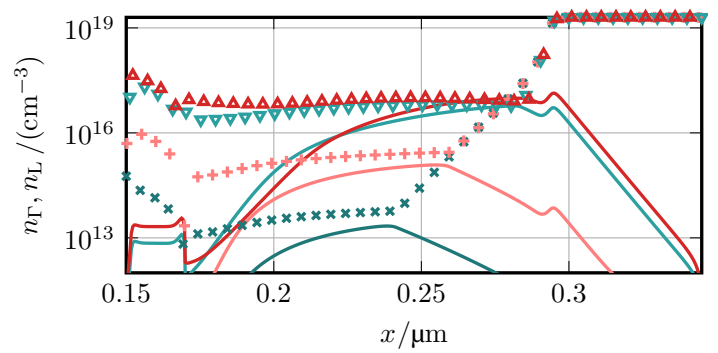

(b)

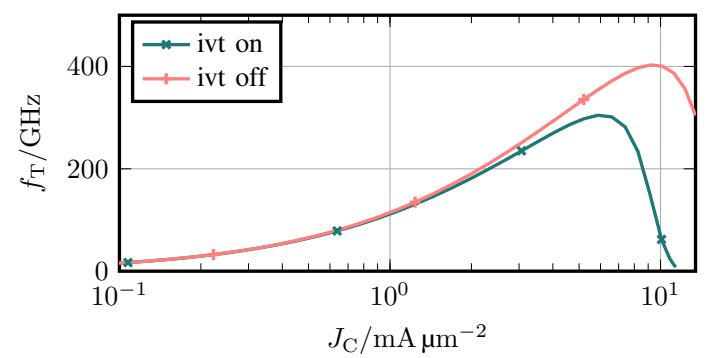

(c)

Fig.9. Simulated $\mathrm{GaAs}_{1-\mathrm{z}} \mathrm{Sb}_{\mathrm{z}} / \mathrm{InP}$ HBT with $z=0.5$ in the base and $z=0$ in all other regions. (a) Band diagram for $V_{\mathrm{BE}}=0 \mathrm{~V}$. (b) Electron density in the $\Gamma$ (solid lines) and L valleys (symbols) and impurity concentration (dashed line) in the base and collector for operating points $J_{\mathrm{C}}=\left[4 \times 10^{-3}, 0.18,4.6,7.4\right] \mathrm{mA} /$ $\mu \mathrm{m}^{2}$. (c) Transit frequency vs. collector current density with intervalley valley transfer turned on and off. $V_{\mathrm{BC}}=-0.2 \mathrm{~V}$ in all cases. The dashed line in (a) indicates the net doping concentration in log scale.

\section{Appendix B: Extracted Model Parameters}

The parameters of the two-valley DD system are listed in the tables below.

Table 1: Parameters for the velocity field model in the $\Gamma$ valley.

\begin{tabular}{|c|c|c|c|c|}
\hline parameter & GaAs & InP & InAs & GaSb \\
\hline \hline$\mu_{\Gamma, \mathrm{L}} /\left(\mathrm{cm}^{2} / \mathrm{Vs}\right)$ & 7060 & 3410 & 23300 & 20300 \\
\hline
\end{tabular}




\begin{tabular}{|l|l|l|l|l|}
\hline \multicolumn{1}{|c|}{ parameter } & \multicolumn{1}{c|}{ GaAs } & \multicolumn{1}{c|}{ InP } & \multicolumn{1}{c|}{ InAs } & \multicolumn{1}{c|}{ GaSb } \\
\hline \hline$\mu_{\Gamma, \min } /\left(\mathrm{cm}^{2} / \mathrm{Vs}\right)$ & 457 & 36 & 324 & 81 \\
\hline$N_{\Gamma, \mathrm{ref}} /\left(10^{17} \mathrm{~cm}^{-3}\right)$ & 0.52 & 1.42 & 0.383 & 0.735 \\
\hline$\alpha_{\Gamma}$ & 0.60 & 0.48 & 0.50 & 0.56 \\
\hline$\beta_{\Gamma}$ & 4.17 & 6.60 & 4.17 & 1.38 \\
\hline$v_{\Gamma, \mathrm{sat}} /\left(10^{7} \mathrm{~cm} / \mathrm{s}\right)$ & 2.30 & 2.35 & 2.60 & 3.76 \\
\hline
\end{tabular}

Table 2: Parameters for the velocity field model in the L valley.

\begin{tabular}{|l|l|l|l|l|}
\hline \multicolumn{1}{|c|}{ parameter } & \multicolumn{1}{c|}{ GaAs } & \multicolumn{1}{c|}{ InP } & \multicolumn{1}{c|}{ InAs } & \multicolumn{1}{c|}{ GaSb } \\
\hline \hline$\mu_{\mathrm{L}, \mathrm{L}} /\left(\mathrm{cm}^{2} / \mathrm{Vs}\right)$ & 377 & 185 & 2240 & 2230 \\
\hline$\mu_{\mathrm{L}, \mathrm{min}} /\left(\mathrm{cm}^{2} / \mathrm{Vs}\right)$ & 62 & 48 & 254 & 103 \\
\hline$N_{\mathrm{L}, \mathrm{ref}} /\left(10^{17} \mathrm{~cm}^{-3}\right)$ & 4.09 & 8.34 & 4.87 & 1.43 \\
\hline$\alpha_{\mathrm{L}}$ & 0.62 & 0.65 & 0.51 & 0.62 \\
\hline$\beta_{\mathrm{L}}$ & 1.73 & 1.98 & 2.27 & 1.98 \\
\hline$v_{\mathrm{L}, \mathrm{sat}} /\left(10^{7} \mathrm{~cm} / \mathrm{s}\right)$ & 0.92 & 0.80 & 1.18 & 0.84 \\
\hline
\end{tabular}

Table 3: Parameters for the intervalley transfer rate model.

\begin{tabular}{|l|l|l|l|l|}
\hline \multicolumn{1}{|c|}{ parameter } & \multicolumn{1}{c|}{ GaAs } & \multicolumn{1}{c|}{ InP } & \multicolumn{1}{c|}{ InAs } & \multicolumn{1}{c|}{ GaSb } \\
\hline \hline$f_{\mathrm{L} \Gamma 0} / \mathrm{THz}$ & 1.70 & 7.99 & 1.59 & 0.11 \\
\hline$\delta_{f} /(\mathrm{GHz} /(\mathrm{kVcm}))$ & 5.79 & 8.96 & 25.0 & 5.33 \\
\hline$a_{\mathrm{f}} / \mathrm{THz}$ & 0.36 & 0.19 & 0.32 & 0.64 \\
\hline$E_{\mathrm{ivt}, 0} /(\mathrm{kV} / \mathrm{cm})$ & 3.00 & 11.4 & 1.63 & 0.019 \\
\hline$N_{\text {ivt }} /\left(10^{16} \mathrm{~cm}^{-3}\right)$ & 78.6 & $1.22 \times 10^{3}$ & 178 & $4.31 \times 10^{-3}$ \\
\hline$\zeta_{\text {ivt }}$ & 0.46 & 0.58 & 0.54 & 0.48 \\
\hline
\end{tabular}

\section{References}

[1] P. Butcher and W. Fawcett, "Intervalley transfer of hot electrons in gallium arsenide", Physics Lett., vol. 17, no. 3, pp. 216-217, 1965.

[2] T. Ishibashi, "Nonequilibrium electron transport in HBTs", IEEE Trans. Electron Dev., vol.48, no. 11, pp. 2595-2605, 2001.

[3] V. Palankovski, S. Selberherr, and R. Schultheis, "Simulation of heterojunction bipolar transistors on Gallium-Arsenide", IEEE Trans. on Electron Dev., vol. 48. pp. 227-230, 1999.

[4] G. Gomila, J. M. Rubi, I. R. Cantalapiedra, and L. L. Bonilla, "Stationary states and phase diagram for a model of the gunn effect under realistic boundary conditions", Physical Rev. E, vol. 56, no. 2, pp. 1490-1499, 1997.

[5] L. L. Bonilla, F. J. Higuera, and S. Venakides, "The Gunn effect: Instability of the steady-state and stability of the solitary wave in long extrinsic semiconductors", SIAM J. on Appl. Mathematics, vol. 54, no. 6, pp. 1521-1541, 1994.
[6] L. P. Tatum, M. Sciullo and M. E. Law, "Simulation of HotElectron Effects with Multi-band Semiconductor Devices," 2018 International Conference on Simulation of Semiconductor Processes and Devices (SISPAD), Austin, TX, 2018, pp. 327-330.

[7] Synopsis Sentaurus Device User Guide, Vers. I, , Dec. 2013.

[8] Minimos-NT, 2.1, Institute for Microelectronics, TU Vienna, 2004.

[9] Genius semiconductor device simulator user guide", 1.7.4, Cogenda, 2009.

[10] H. Shao and P. Zampardi, "What silicon modelers should know about III-Vs for TCAD", 3rd MOS-AK workshop, Beijing, June 2018.

[11] G. Wedel, T. Nardmann, and M. Schroter, "On the use of Drift-Diffusion and Hydrodynamic Transport Models for Simulating the Negative Differential Mobility Effect", IEEE BiCMOS and Compound Semicond. Integr. Circ. and Technol. Symp. (BCICTS), San Diego (CA), USA 2018.

[12] J. C. Li, M. Sokolich, T. Hussain, and P. M. Asbeck, "Physical modeling of degenerately doped compound semiconductors for high-performance HBT design", SolidState Electronics, vol. 50, no. 7-8, pp. 1440-1449, 2006.

[13] P. J. Zampardi and F. Kharabi, "Industrial view of III-V devices compact modeling for circuit design", IEEE Trans. Electron Dev., vol. 66, no. 1, pp. 28-33, 2019.

[14] M. Schroter, T. Nardmann, and G. Wedel, "A Closed Form Solution for the Low-Current Collector Transit Time in Group IV and Group III-V HBTs", IEEE Trans.Electron Dev., vol. 64, no. 8, pp. 3346-3352, 2017.

[15] K.Blotekjaer,"Transport equations for electrons in two valley semiconductors", IEEE Trans. Electron Dev., vol. 17, no. 1 , pp. $38-47,1970$.

[16] K. Tomizawa, Numerical Simulation of Submicron Semiconductor Devices. Artech House, 1993.

[17] G. Wedel, A Box-Integration/WENO solver for the Boltzmann Transport Equation its Application to HighSpeed Heterojunction Bipolar Transistors. Dissertation, CEDIC, TU Dresden, 2017.

[18] S.-M. Hong, A.-T. Pham, C. Jungemann, "Deterministic solvers for the Boltzmann transport equation", Springer, WienNewYork, 2011.

[19] X. Wen, A. Arabhavi, O. Ostinelli, C. Bolognesi, T. Zimmer, C. Maneux, M. Luisier, C. Mukherjee, C. Raya, B. Ardouin, M. Deng, S. Fregonese, V. Nodjiadjim, M. Riet, and W. Quan, "A multiscale TCAD approach for the simulation of InP DHBTs and the extraction of their transit times", IEEE Trans. Electron Dev., vol. 66, no. 12, pp. 50845090, 2019.

[20] M. Lundstrom, "Carrier transport in BJTs: From ballistic to diffusive and off-equilibrium", IEEE BiCMOS and Compound Semicond. Integr. Circ. and Technol. Symp. (BCICTS), San Diego (CA, USA), 2018.

[21] M. Schröter and A. Chakravorty, Compact Hierarchical Bipolar Transistor Modeling With HiCUM. World Scientific, 2010. 
[22] Y. S. Chauhan, S. Venugopalan, M. A. Karim, S. Khandelwal, N. Paydavosi, P. Thakur, A. M. Niknejad and C. C. Hu, "BSIM Industry standard compact MOSFET models", Europ. Solid-St. Circ. Conf., pp. pp. 30-33, 2012.

[23] K. Tomizawa and D. Pavlidis, "Transport equation approach for heterojunction bipolar transistors", IEEE Trans. on Electron Dev., vol. 37, no. 3, pp. 519-529, 1990.

[24] M. Claus, S. Mothes, S. Blawid, and M. Schröter, "COOS: A wave-function based Schrödinger-Poisson solver for ballistic nanotube transistors", J. of Comp. Electronics, vol. 13, no. 3, pp. 689-700, 2014.

[25] J. S. Blakemore, "Semiconducting and other major properties of gallium arsenide", J. of Appl. Phys., vol. 53, no. 10, R123-R181, 1982.

[26] W.Fawcett, D.C. Herbert "High-field transport in indium phosphide", Electron. Lett., vol.9, no. 14, 308-309, 1973.

[27] T. Grasser, T.-W. Tang, H. Kosina, and S. Selberherr,"A review of hydrodynamic and energy-transport models for semiconductor device simulation", Proc. of the IEEE, vol. 91, no. 2, pp. 251-274, 2003.

[28] M. Schröter, T. Rosenbaum, P. Chevalier, B. Heinemann, S. P. Voinigescu, E. Preisler, J. Bock, and A. Mukherjee, "SiGe HBT technology: Future trends and TCAD-based roadmap", Proc. of the IEEE, vol. 105, no. 6, pp. 1068-1086, 2017.

[29] J. Korn, Device simulation of high-performance SiGe heterojunction bipolar transistors. Diss., TU-Berlin, 2018.

[30] A. H. Marshak and K. M. van Vliet, "Electrical current in solids with position-dependent band structure", Solid-State Electron., vol. 21, no. 2, pp. 417-427, 1978.

[31] M. Lundstrom, Fundamentals of Carrier Transport, Cambridge University Press, 2000.

[32] H. Arabshahi, M. R. Khalvati, and M. R. Rokn-Abadi, "Temperature and doping dependencies of electron mobility in InAs, AlAs and AlGaAs at high electric field application", Brazilian J. of Phys., vol. 38, no. 3a, 2008.

[33] T. J. Maloney and J. Frey, "Transient and steady-state electron transport properties of GaAs and InP,", J. of Appl. Phys., vol. 48, no. 2, pp. 781-787, 1977.

[34] D. Caughey and R. Thomas, "Carrier mobilities in silicon empirically related to doping and field", Proc. of the IEEE, vol. 55, no. 12, pp. 2192-2193, 1967.

[35] D.E. McCumber and A.G. Chynoweth, "Theory of negative-conductance and of Gunn instabilities in two-valley semiconductors", IEEE Trans. Electron. Dev. vol. ED-13, no. 1, pp. 4-21, 1966

[36] B.Gokhale,"Numerical solutions for a one -dimensional silicon n-p-n transistor", IEEE Trans. Electron Dev. vol. 17, no. 8, pp. 594-602, 1970.

[37] J. W. Slotboom, G. Streutker, M. J. van Dort, P. H. Woerlee, A. Pruijmboom and D. J. Gravesteijn, "Non-local impact ionization in silicon devices", Int'l Electron Dev. Meeting, Washington, DC, USA, pp. 127-130, 1991.

[38] R. Lövblom, Developement of Sub-Millimeter-Wave Inp/ GaAsSb Double Heterojunction Bipolar Transistors.
Dissertation, Lund University, 2014.

[39] V. Palanovski, Simulation of Heterojunction Bipolar Transistors. Diss., TU-Wien, 2000.

[40] M. Schröter, A compact physics-based large-signal modelfor high-speed bipolar transistors with special consideration of high current densities and two-dimensional effects, Diss. (in German), AG Halbleiterbauel., RuhrUniversität Bochum, Germany, 1987.

[41] G. Wachutka, "Unified framework for thermal, electrical, magnetic, and optical semiconductor device modeling", COMPEL, Vol. 10, No. 4, pp. 311-321, 1991.

[42] D. Chang and J. G. Fossum, "Simplified energy-balance model for pragmatic multi-dimensional device simulation", Solid-State Electronics, vol. 41, no. 11, pp. 1795-1802, 1997. 